Thorax, 1978, 33, 175-180

\title{
Lung injury following a 50-metre fall into water
}

\author{
H. THOMAS ROBERTSON, S. LAKSHMINARAYAN, AND LEONARD D. HUDSON \\ From the Department of Medicine, University of Washington School of Medicine, University of \\ Washington, Seattle, Washington 98195, USA
}

Robertson, H. T., Lakshminarayan, S., and Hudson, L. D. (1978). Thorax, 33, 175-180. Lung injury following a 50-metre fall into water. The pulmonary complications of a 50-metre fall to the water (a form of suicide attempt producing $87 \%$ mortality) were studied in 15 survivors. Presenting findings included crackles, haemoptysis, and hypotension. The alveolar-arterial oxygen difference was greater than $150 \mathrm{mmHg}(20 \mathrm{kPa})$ in nine subjects on admission. Ventilatory failure developed in 10 of the patients, including all of those with massive haemoptysis. Radiographic findings included pneumothorax and diffuse pulmonary opacities adjacent to the area of impact. Pneumothorax developed within 12 hours of admission in 10 of 15 subjects but was associated with rib fractures in only four subjects. The clinical course of the condition is consistent with the hypothesis that the traumatic pulmonary tears produced interstitial emphysema, with subsequent development of pneumomediastinum, subcutaneous emphysema, and pneumothorax. Pneumothorax is a common complication of severe lung contusion even in the absence of penetrating pleural injury.

Severe lung contusion, resulting in diffuse alveolar haemorrhage and impaired pulmonary gas exchange, has been described in many forms of blunt and penetrating thoracic trauma. Of these, pulmonary blast injury has been well studied in humans (Falla, 1940; Hadfield et al., 1940; Wilson and Tunbridge, 1943). Early experimental studies (Zuckerman, 1940) demonstrated that pulmonary injury is due to the direct impact of the blast wave and hence equivalent to any other form of blunt lung trauma. In human blast trauma victims, the lungs contained large quantities of intra-alveolar blood, and neither rib fractures nor pneumothorax were described. The external manifestations of injury were limited to the finding of frothy bloodstained sputum at the mouth and nostrils.

Pneumothorax is associated with blunt chest injury, but as all the recent clinical reviews of this entity deal primarily with car accident victims with direct injury to the bony thoracic cage (Alfano and Hale, 1965; DeMuth and Smith, 1965; Hankins et al., 1973; Roscher et al., 1974) it is difficult to assess the importance of lung contusion alone in the development of barotrauma. We have had the unique opportunity to study the clinical course of 15 attempted suicides leaping to the water from Seattle's Aurora Bridge, a major traffic arterial HL 19457 and HL 05819 that is 50 metres above the water at mid-span. This form of blunt trauma is often uncomplicated by rib fracture or other penetrating injury and offers an opportunity to examine the relationship between uncomplicated lung contusion and barotrauma.

\section{Methods}

The number of suicide attempts was obtained from the files of a Seattle newspaper, which keeps a tally of persons known to have jumped from the Aurora Bridge. Since the completion of the bridge in 1934, 142 persons are known to have landed in the water, those missing the water being excluded from this study. The newspaper data on fatalities over the past nine years were cross-checked with the records of the King County coroner. Of the 18 known survivors, the first was a high diver by avocation, who ostensibly made his leap without suicidal intent, wearing a football helmet and protective kapok vest. The remaining 17 subjects jumped with suicidal intent and were admitted to hospital. Complete medical records were available on the 15 survivors since 1961 , and these patients comprise this account. All eight survivors seen since 1974 were followed by one of us, while the remaining seven were studied by review of their records. Only one patient (No. 3), a smoker with 


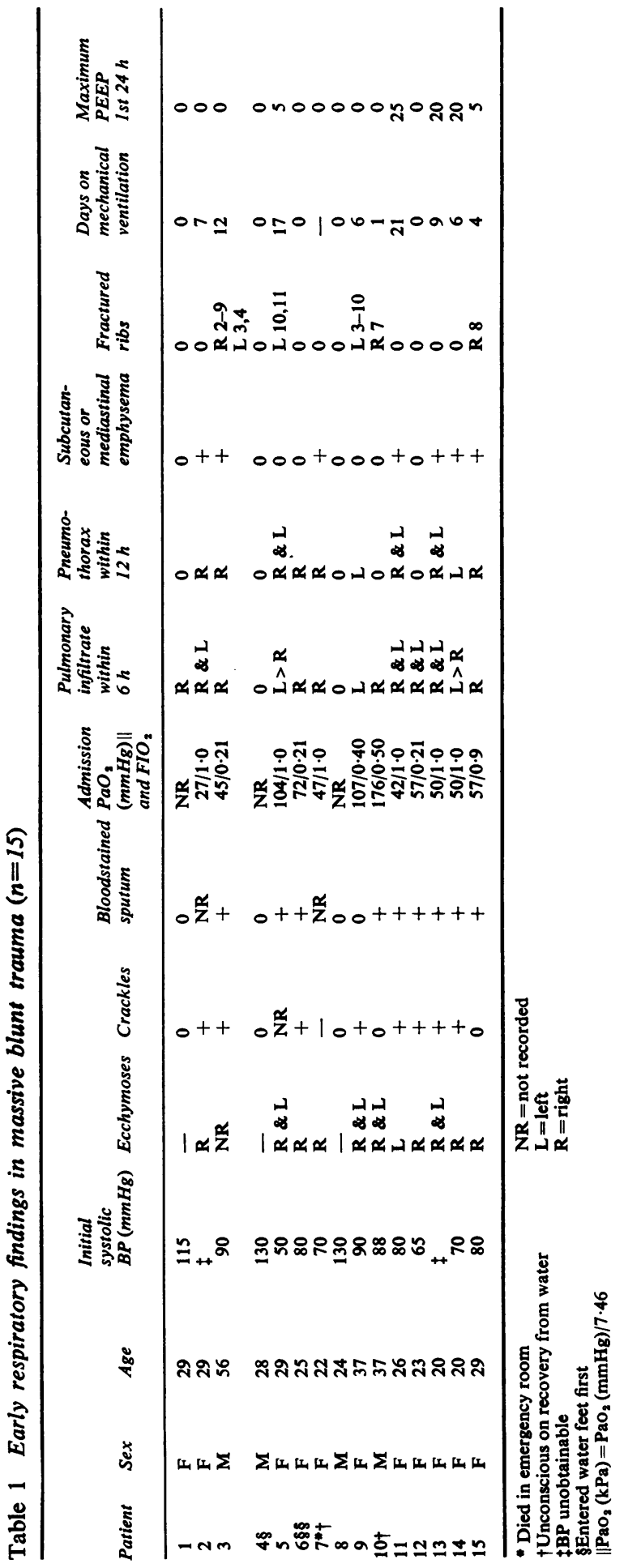


chronic productive cough, had a previous history of pulmonary symptoms. All chest radiographs were interpreted by the three authors separately, and in cases of disagreement the majority opinion was applied. The first two patients requiring ventilatory assistance were treated with a pressurecycled ventilator, and the remainder with a volume-cycled ventilator with tidal volumes of 10 to $15 \mathrm{ml} / \mathrm{kg}$.

\section{Results}

Of the 124 fatalities, 83 were men and 41 were women, with a median age of 39 (range 20-83) years. Of the 18 survivors, five were men and 13 women, with a median age of 28 (range 20-56) years. Youth correlated with survival (Wilcoxon test, $P<0.01)$, and female sex was associated with survival $\left(\chi^{2}, P<0.01\right)$.

Two of the 15 subjects of the study (patients 4 and 6, Table 1) had been observed to enter the water cleanly, feet first, and did not incur substantial injury. Two of the subjects (patients 7 and 10) were unconscious at the time they were removed from the water. As there is heavy pleasure boat traffic beneath the bridge and a nearby harbour patrol station, the estimated time in the water before rescue was brief, ranging from less than one minute to 10 minutes. Rapid clinical deterioration with shock and respiratory distress was noted in all but three (patients 1, 4, and 6) of the 15 subjects by the receiving paramedical squad at the harbour patrol dock. Five subjects required endotracheal intubation and nine required intravenous fluids for treatment of hypotension before transport.

The presenting clinical signs in the 15 patients are shown in Table 1 . The characteristic physical findings were ecchymoses over the area of impact (12 of 15 subjects), crackles (10 of 14 subjects), and the production of frothy bloodstained sputum ( 9 of 13 subjects). An alveolar-arterial $\mathrm{O}_{2}$ difference of more than $150 \mathrm{mmHg}(20 \mathrm{kPa})$ was observed in nine of the 12 patients who had arterial blood gases measured on admission. The inspired oxygen fraction for this measurement is noted in Table 1 . Metabolic acidosis, as manifested by an anion gap of greater than $15 \mathrm{mEq} / 1$ was seen in 10 of 12 subjects, including all those with a systolic blood pressure of less than $90 \mathrm{mmHg}$.

The commonest radiological abnormality was pulmonary opacities of the acinar filling type. These appeared within six hours of injury and were seen in 13 patients (see Table 1). The radiological abnormalities were noted to be underlying the areas of external ecchymoses seen on physical examination. Radiological evidence of pneumothorax was present within 12 hours of injury in 10 patients, and was bilateral in three. Rib fractures were found in initial and follow-up films only in the five oldest subjects, one of whom did not develop pneumothorax. Hence pneumothorax without rib fracture developed within the first 12 hours in six patients. Subcutaneous emphysema was noted in five patients before the insertion of chest tubes, and was recorded in two before pneumothorax occurred. Pneumomediastinum was seen on presentation in four patients.

One patient died in the emergency room of severe shock and hypoxaemia. Of the 14 long-term survivors, nine required assisted ventilation for one to 21 (mean 7.9) days, and three required positive end expiratory pressure of $20 \mathrm{~cm} \mathrm{H} \mathrm{H}_{2} \mathrm{O}$ or more to maintain a $\mathrm{PaO}_{2}$ greater than $60 \mathrm{mmHg}$ $(8 \mathrm{kPa})$ breathing $50 \%$ oxygen. For the nine longterm survivors with pneumothorax, chest tubes were required for 4 to 21 (mean 8.6) days. Eight of the nine required mechanical ventilation. The pneumothorax group was characterised by prolonged air leaks from the thoracostomy tubes (mean 8.7, range 2-21 days), most prominent in those with severe respiratory failure. Three patients had recurrent pneumothoraces after the tubes had been removed. Three patients with severe bilateral air leaks were bronchoscoped within 24 hours of presentation to exclude bronchial laceration. No tears were noted, but petechial submucosal haemorrhages were seen in the bronchi of contused segments in two of the three patients. No subsequent evidence of postlaceration bronchial stenosis was seen in any patient. Vigorous haemorrhage welling up from the small airways was observed by bronchoscopy in three of the five most hypoxaemic subjects, including one who produced over $900 \mathrm{ml}$ of blood in the first 12 hours.

The other injuries incurred included visceral lacerations and fractures (Table 2). Haematuria was recorded in nine of the 15 patients, all of

Table 2 Associated injuries in massive blunt trauma

\begin{tabular}{lll}
\hline $\begin{array}{l}\text { Laparotomy required } n=5 \\
\quad \text { Ruptured spleen } \\
\text { Liver lacerations }\end{array}$ & 5 & \\
Bowel lacerations & 4 & $(5,8,9,10,11)$ \\
Fractures and dislocations $n=8$ & 2 & $(8,9,10,11)$ \\
Pelvis & 3 & $(10,11)$ \\
Skull & 2 & \\
Neck & 1 & $(1,5,10)$ \\
Clavicle & 1 & $(2,10)$ \\
Femur & 1 & $(14)$ \\
Hip dislocations & 1 & $(8)$ \\
\end{tabular}

Numbers in parentheses refer to patient numbers in Table 1. 
whom had normal intravenous pyelograms and no subsequent evidence of urological injury. In the one to 16 years of follow-up in this patient group, there has been only one recorded subsequent suicide attempt.

In the past four years, all bridge leapers who were dead at the time of recovery from the water have had postmortem examinations performed by the King County medical examiner, a forensic pathologist. The primary mechanism of death was considered to be a massive internal haemorrhage from visceral lacerations. Lung contusions, generally accompanied by rib fractures, have been observed. Pneumothorax has not. been noted in any of the fatalities, including those of the past year in which this was specifically sought as a mechanism of death. Aortic disruption has not been seen in this group.

\section{Discussion}

The circumstances of the injury, the haemoptysis, and the location of the major radiographic opacities beneath the area of ecchymosis on the skin suggest that the primary injury in these patients was lung contusion. Experimental studies of lung contusion have also shown that the primary lesion is beneath the area of impact (Rutherford and Valenta, 1971; Trinkle et al., 1973), though not necessarily limited to that spot. The maximum speed at the end of a 50-metre free fall would be approximately $115 \mathrm{~km} / \mathrm{h}$, and it has been demonstrated that extensive lung contusions can be produced by the same mechanism in guinea-pigs striking the water at a slightly slower entry speed (Stewart et al., 1955). Thus, the trauma incurred by the patients was of sufficient violence to produce lung contusion, and the finding of a frothy bloodstained sputum further supports the diagnosis. The finding of submucosal bronchial haemorrhage in the three subjects who underwent bronchoscopy has also been seen in blast injury fatalities (Falla, 1940), another form of contusion injury.

The striking observation in this patient group was that of the 10 subjects with pneumothorax, only four had rib fractures. Rupture of visceral pleura is the usual mechanism evoked to explain traumatic pneumothorax, but such lacerations in the absence of rib fractures have not been described in lethal blast injury to animals, nor have they been noted in necropsy studies of human blast injury victims. However, the studies of barotrauma by Macklin (1937) provide a plausible explanation for the clinical findings presented. Macklin noted that when lungs of experimental animals were subjected to sufficient inflation pressures to produce parenchymal tears, the lung? did not rupture at their visceral surface, bue pneumothorax still occurred. The air leaks from? the torn alveolar units were shown to track back to the hilum along the pulmonary vessels. Onces at the hilum, the air spread beneath the thinmediastinal pleura, whence it tracked both behind the peritoneum and into the subcutaneous tissues $\vec{\omega}$ When pneumothorax occurred, it was a result of rupture of the distended mediastinal pleura. The $\vec{x}$ hyperinflation mechanism for the production of w pulmonary parenchymal lacerations with subse quent mediastinal emphysema has been suspectedin several clinical situations. The fascial planesr permitting the passage of fluid surrounding pul으 monary vessels into the mediastinum have beendemonstrated in human cadavar studie 8 (Marchand, 1951). Infants resuscitated from: respiratory distress syndrome (Campbell, 1970) $\overrightarrow{0}$ divers with barotrauma (Edmonds, 1976), patients with severe asthma (Karetsky, 1975), and patients with early adult respiratory distress syndromeo (ARDS) subjected to extremely high tidal volumess (Bone et al., 1978) all have been noted to develop? pneumothorax after pneumomediastinum. $A \frac{0}{D}$ recent pathological and radiological study of adulf respiratory distress patients (Wescott and Cole $\overrightarrow{\overrightarrow{0}}$ 1974) showed interstitial air surrounding the pul 3 monary vessels in association with pneumo mediastinum.

The hyperinflation mechanisms cited above are thought to produce pulmonary parenchymafof lacerations, which in turn lead to the barotrauma $\times$ Part of the contusion injury includes parenchyma lacerations, which could lead to barotrauma by the same mechanism. Thus, in patients with such contusions, as in those with hyperinflation injury the passage of air along these pulmonary vascularplanes is facilitated by negative pressure in the mediastinum relative to the alveolus, and by the forces acting between the distensible pulmonary tissue and the less distensible pulmonary vascula ture (Mead et al., 1970). This hypothesis woulch explain the slow expression of the pneumothoraces $\omega$ seen in the survivors and the absence of pneumo thorax in the fatalities, as continued respiratorye effort after the injury would be required to allowe air to track to the mediastinum and eventually rupture the mediastinal pleura. This would alsoexplain the prolonged air leaks noted in our sub $\overrightarrow{\mathrm{D}}$ jects. There is some experimental evidence that supports this mechanism of barotrauma after lung contusion. Macklin and Macklin (1944) noted that in a published account of experimental blast in $\delta$ jury where the animals survived the initial injuryo 
mediastinal emphysema was demonstrated (Zuckerman, 1940), though this was not commented on by the author.

The radiological literature maintains a distinction between lung contusion, defined by immediate onset of diffuse alveolar haemorrhage which clears in four to six days, and lung laceration, which presents identically but forms a haematoma and/ or cysts, which persist for many months (Williams and Stembridge, 1964; Stevens and Templeton, 1965; Wiot, 1975). By this definition, 12 of our 15 patients had both contusion and laceration. This distinction seems trivial since both injuries have an identical pathogenesis, differing only in extent.

No acute or chronic evidence of bronchial tear was noted in any patient in this series. Traumatic bronchial laceration usually results from direct trauma to the sternal area, and the tears described in most series are in extrapulmonary airways (Reynolds and Davis, 1966; Eastridge et al., 1970). Laceration of bronchi does not appear to have been described in the literature on experimental and human blast injury. Thus, even though diffuse blunt chest injury can produce severe air leaks, these are not necessarily secondary to bronchial laceration.

Systemic air embolism has been observed in experimental blast injury (Clemedson, 1956). As a consequence of this, some authors have advocated avoiding mechanical ventilation whenever possible in patients with lung contusion, minimising deep inspiratory manoeuvres, and maintaining a headdown position (Ratliff et al., 1971). However, this therapeutic approach was associated with a significant mortality rate from acute respiratory failure. Although systemic air embolism remains a theoretical complication of mechanical ventilation in patients with pulmonary tears, it did not occur in any of our patients.

It is noteworthy that in these patients the severity of the respiratory failure was quite striking. Four would have met the criteria for inclusion in the National Cooperative Membrane Oxygenator Study (Blake, 1976). The fact that 14 of the 15 victims survived emphasised the reversible nature of this form of respiratory failure. The hypoxaemia, severe air leaks, and massive alveolar bleeding can be managed successfully with current techniques of ventilatory care.

\section{References}

Alfano, G. S., and Hale, H. W. (1965). Pulmonary contusion. Journal of Trauma, 5, 647-658.

Blake, L. H. (1976). Goals and progress of the National Heart and Lung Institute collaborative extra- corporeal membrane oxygenation study. In Artificial Lungs for Acute Respiratory Failure, edited by W. Zapol and J. M. Qvist, pp. 513-524. Academic Press, New York.

Bone, R. C., Francis, P. B., and Pierce, A. K. (1978). Pulmonary barotrauma complicating positive end expiratory pressure. American Review of Respiratory Disease. (In press).

Campbell, R. E. (1970). Intrapulmonary interstitial emphysema: A complication of hyaline membrane disease. American Journal of Roentgenology, Radium Therapy and Nuclear Medicine, 110, 449456.

Clemedson, C. (1956). Blast injury. Physiological Reviews, 36, 336-354.

DeMuth, W. E., Jr., and Smith, J. M. (1965). Pulmonary contusion. American Journal of Surgery, 109, 819-823.

Eastridge, C. E., Hughes, F. A., Jr., Pate, J. W., Cole, F., and Richardson, R. (1970). Tracheobronchial injury caused by blunt trauma. American Review of Respiratory Disease, 101, 230-237.

Edmonds, C. (1976). Barotrauma. In Diving Medicine, edited by R. H. Strauss, pp. 54-59. Grune and Stratton, New York.

Falla, S. T. (1940). Effect of explosion-blast on the lungs: report of a case. British Medical Journal, 2, 255-256.

Hadfield, G., Swain, R. H. A., Ross, J. M., and Drury-White, J. M. (1940). Blast from high explosive: preliminary report on ten fatal cases. Lancet, 2, 478-481.

Hankins, J. R., Attar, S., Turney, S., Cowley, R. A., and McLaughlin, J. S. (1973). Differential diagnosis of pulmonary parenchymal changes in thoracic trauma. American Surgeon, 39, 309-318.

Karetsky, M. S. (1975). Asthma mortality: an analysis of one year's experience, review of the literature, and assessment of current modes of therapy. Medicine, 54, 471-484.

Macklin, C. C. (1937). Pneumothorax with massive collapse from experimental local over-inflation of the lung substance. Canadian Medical Association Journal, 36, 414-420.

Macklin, M. T., and Macklin, C. C. (1944). Malignant interstitial emphysema of the lungs and mediastinum as an important occult complication in many respiratory diseases and other conditions: An interpretation of the clinical literature in the light of laboratory experiment. Medicine, 23, 281-358.

Marchand, P. (1951). The anatomy and applied anatomy of the mediastinal fascia. Thorax, 6, 359368.

Mead, J., Takishima, T., and Leith, D. (1970). Stress distribution in the lungs: a model of pulmonary elasticity. Journal of Applied Physiology, 28, 596608.

Ratliff, J. L., Fletcher, J. R., Kopriva, C. J., Atkins, C., and Aussem, J. W. (1971). Pulmonary contusion: a continuing management problem. Journal of Thoracic and Cardiovascular Surgery, 62, 638-644. 
Reynolds, J., and Davis, J. T. (1966). Injuries of the chest wall, pleura, pericardium, lungs, bronchi and esophagus. Radiological Clinics of North America, 4, 383-401.

Roscher, R., Bittner, R., and Stockmann, U. (1974). Pulmonary contusion: clinical experience. Archives of Surgery, 109, 508-510.

Rutherford, R. B., and Valenta, J. (1971). An experimental study of 'traumatic wet lung'. Journal of Trauma, 11, 146-166.

Stevens, E., and Templeton, A. W. (1965). Traumatic nonpenetrating lung contusion. Radiology, 85, 247252.

Stewart, W. K., Spells, K. E., and Armstrong, J. A. (1955). Lung injury by impact with a water surface. Nature, 175, 504-505.

Trinkle, J. K., Furman, R. W., Hinshaw, M. A., Bryant, L. R., and Griffen, W. O. (1973). Pulmonary contusion: Pathogenesis and effect of various resuscitative measures. Annals of Thoracic Surgery, 16, 568-573.
Wescott, J. L., and Cole, S. R. (1974). Interstitial $\overrightarrow{\vec{F}}$ pulmonary emphysema in children and adults: roentgenographic features. Radiology, 111, 367378.

Williams, J. R., and Stembridge, V. A. (1964). Pul- $\frac{\widehat{D}}{\Phi}$ monary contusion secondary to nonpenetrating $\bigcirc$ chest trauma. American Journal of Roentgenology, $⿻$ Radium Therapy and Nuclear Medicine, 91, 284- $\vec{\circ}$ 290.

Wilson, J. V., and Tunbridge, R. E. (1943). Patho- $\vec{\omega}$ logical findings in a series of blast injuries. Lancet, $\stackrel{\omega}{\omega}$ 1, 257-261.

Wiot, J. (1975). The radiologic manifestations of blunt ${ }^{x}$ chest trauma. Journal of the American Medical $\omega_{\omega}^{\omega}$ Association, 231, 500-503.

Zuckerman, S. (1940). Experimental study of blast injuries to the lungs. Lancet, 2, 219-224.

Requests for reprints to: Dr. H. T. Robertson, Divisionof Respiratory Disease, RM-12, University Hospital, $>$ Seattle, Washington 98195, USA. 